\title{
Freedom-7: A High Fidelity Seven Axis Haptic Device With Application To Surgical Training
}

\author{
V. Hayward, P. Gregorio, O. Astley, S. Greenish, M. Doyon \\ Dept. of Electrical Engineering and Center for Intelligent Machines \\ McGill University, Montréal, Canada \\ http://www.cim.mcgill.ca/ hayward/Home.html \\ L. Lessard, J. McDougall \\ Dept. of Mechanical Engineering \\ McGill University, Montréal, Canada \\ http://www.mecheng.mcgill.ca/〜 zen/Home.html \\ I. Sinclair, S. Boelen, X. Chen, J.-P. Demers, J. Poulin, \\ I. Benguigui, N. Almey, B. Makuc, X. Zhang \\ MPB Technologies Inc., Pointe Claire, Canada \\ http://www.total.net/ mpbt \\ DRAFT PAPER: PREPRINTS OF ISER'97, June 15-18 1997, Barcelona Spain.

\begin{abstract}
A seven axis haptic device, called the Freedom-7, is described in relation to its application to surgical training. The generality of its concept makes it also relevant to most other haptic applications. The design rationale is driven by a long list of requirements since such a device is meant to interact with the human hand: uniform response, balanced inertial properties, static balancing, low inertia, high frequency response, high resolution, low friction, arbitrary reorientation, and low visual intrusion. Some basic performance figures are also reported.
\end{abstract}

\section{Introduction}

It is suggested that the future of surgical simulation for training [1] will follow a path similar to that of flight simulation for aviation training [2], which has now become an industry justifying significant research in a plurality of domains.

We describe an electromechanical device capable of supporting the simulation of tasks carried out with a variety of surgical instruments including, knives, forceps, scissors, and micro-scissors. In these categories, individual instruments differ from each other by their business-end and by the interface they present to the human hand. What most of these instruments have in common are seven degrees of kinematic freedom, with the exception of the knife, which has only six. The device incorporates a mechanical interface which enables the interchange of handles, for example to support these categories of instruments 
and to provide the force feedback needed to simulate the interaction of the instrument with a tissue. The Freedom-7 is described in relation to surgical simulation, but its general purpose design makes it relevant to most haptic applications: multi-dimensional data set exploitation, teleoperation, computer aided design and animation, and so-on. The present paper is a follow-up to [3]

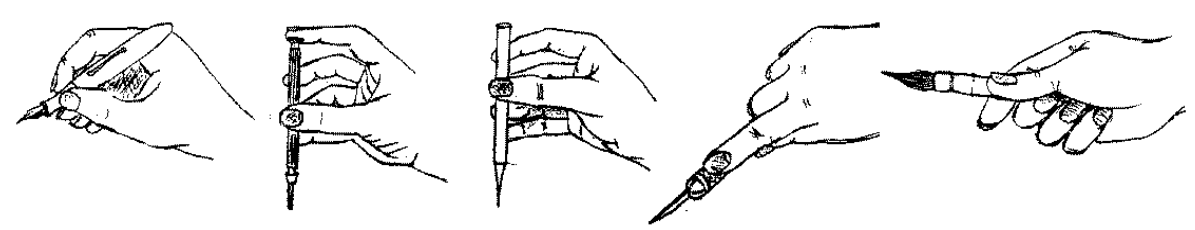

Figure 1. Five ways humans manipulate small handles.

where much of the motivation is discussed. It is argued that there is considerable motivation for the development of devices designed to support the haptic emulation of tasks involving small tools: pens, styli, brushes, screwdrivers, cutter knives, lancets, scapels, and so-on, see Figure 1. In all these cases, a person is gripping a small handle which is free at one end, while the other marks the location of the task where the tool interacts with an object. The handle proximal end must be free of attachments for realism of the simulation given the great variety of hand configuration and re-configurations. Surgical instruments are no exception to this.

In the present paper we focus on the design of the Freedom- 7 as it relates to instruments selected from the basic dissection set illustrated in Figure 2. Some basic performance figures are also reported and discussed.

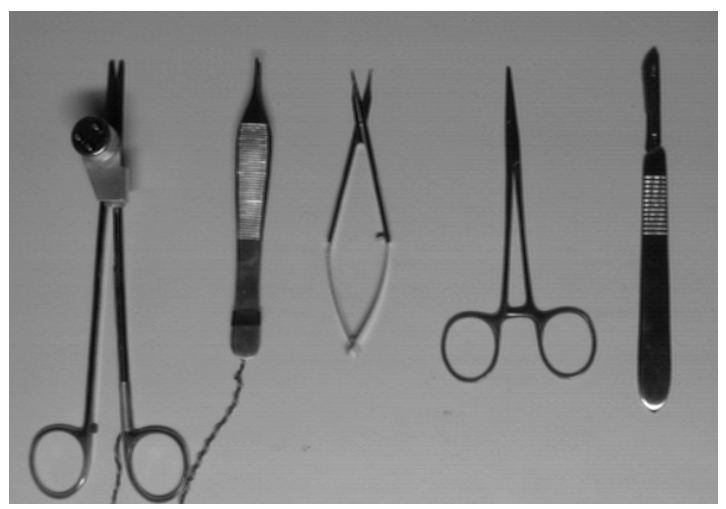

Figure 2. Five families of instruments: scissors (please ignore the attachment), tissue forceps, micro-scissors, hemostatic forceps, and knife.

\section{The Basic Dissection Kit}

Referring to Figure 2, from left to right we find scissors, a tissue forceps, microscissors, a hemostatic forceps, and a knife. Actual sets contains families of instruments in these category [4]. For example, scissors may come in various 
lengths and sturdiness; forceps can be curved, have teeth, etc. The selected subset nevertheless represents most encountered structural characteristics.
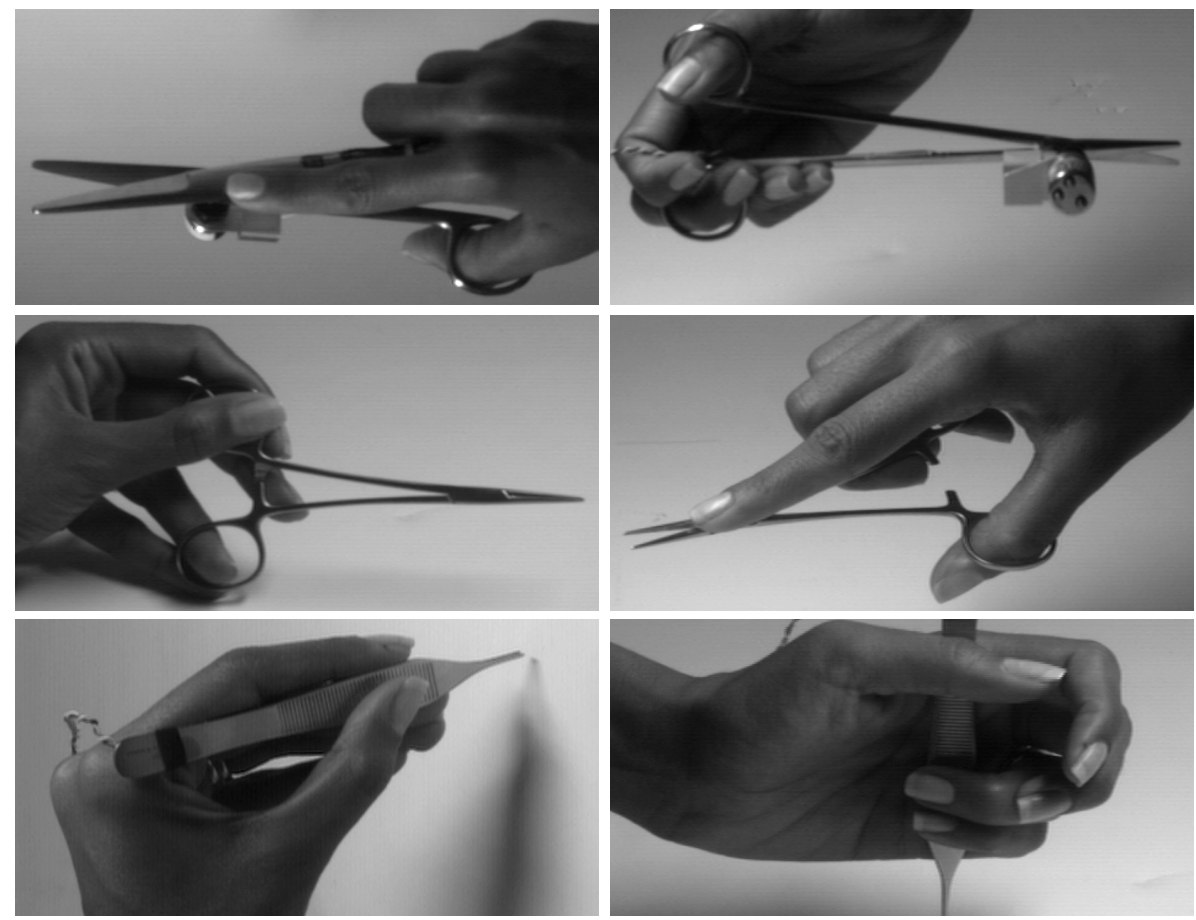

Figure 3. Various ways of holding scissors and forceps.

\subsection{What These Instruments Have in Common}

For purposes of simulation, it should be possible to position and orient these instruments in the greatest achievable work volume. Forces and torques should also be reflected with a sufficient amount of fidelity. They all locate the task at one extremity, but the way they are held may vary greatly. Scissors, for example, may be held in drastically different poses. Forceps and knives are also subject to a diversity of holding positions. See Figure 3.

\subsection{How They Differ}

The knife category requires six degrees of freedom, but all the others involve a closing action which requires a seventh freedom with force feedback. Instruments differ by the interface they present to the hand. They also greatly differ from the view point of their own internal dynamics. For example, tissue forceps and micro-scissors can be represented by spring dynamics. Scissors have instead dissipative dynamics due to friction occurring at the hinge and between blades, introducing high frequency textural sensations. Hemostatic forceps have complicated potential and dissipative dynamics when the ratchet is engaged or disengaged. With scissors and hemostatic forceps, internal dynamics can create high level of forces. 
The approach was to design the device to deliver three forces and three torques with respect to the ground, plus one differential force available at the tip. The device includes a quick attachment interface to accommodate actual instruments. For all closing instruments, one branch is connected to a flange where forces and torques are available, while the other is attached to a sliding rod to provide for differential force feedback. With this approach, the haptic device is only responsible for producing forces and torques resulting from the interaction of an instrument with a tissue which are combined with the natural dynamics of each instrument. In the case of knives, the differential flange is constrained so the device becomes a six axis haptic device with improved performance due to actuator redundancy [5].

\section{Design Rationale}

A haptic device is a two-way transducer to address the human hand motor and sensorial capabilities. By analogy with any transducer designed to stimulate human senses, it is believed that there is more to be gained from wide dynamic range and high resolution than from the sheer amplitude of the transduced signals (force, position and derivatives) in terms of realism and intelligibility.

Throughout the history of master arm designs $[6,7,8]$, direct drive manipulators $[9,10]$, and now haptic devices [11], static balancing is viewed as a major design goal. One motivation is to fully allocate actuator torques to the generation of acceleration. The maximization of acceleration capability was found to be a major factor of performance for haptic devices [12]. Static balancing satisfies one additional crucial requirement for the Freedom-7. Due the diversity of the hand positions that must be accommodated, the device must be easily re-oriented and must preserve its entire set of dynamic properties under re-orientation. Because haptic devices should be general purpose transducers, a uniform response as well as balanced inertial properties (uniform principal inertia terms and minimized coupling terms) must also be included in the list of requirements. The need for a high frequency response imposes severe constraints on the structural design and the elimination of transmission backlash. Absence of friction is needed for output dynamics range. Large work-space and minimum intrusion in the operator's visual and manipulation space must be achieved. We set out to meet these requirements for a total of seven degrees of freedom, while minimizing the complexity of the construction and maintenance.

Many six degrees of freedom parallel linkages were examined, but none were found to meet a reasonable subset of the above requirements (lack of angular workspace, no balancing, heavily coupled inertial tensor, too many joints, bulk and intrusion). Purely serial designs could satisfy even fewer of these requirements. Consequently, a hybrid design was developed. It consists of a distal orienting-plus-sliding stage with a parallel structure supported by a three degree-of-freedom positioning stage including a four-bar mechanism, a structure commonly found in manipulator design. In both cases, the merits of each structure have been best taken advantage of to optimize the system as a whole. The position stage is directly driven, but the orienting stage has 


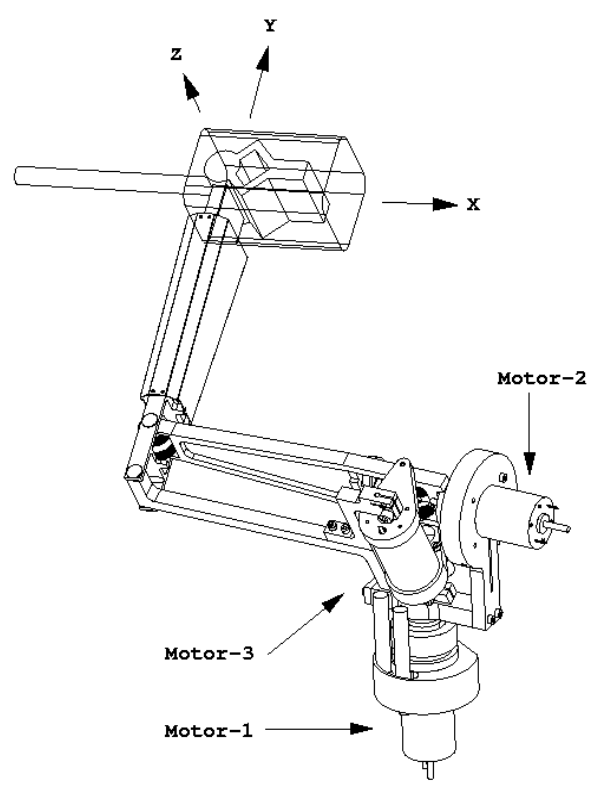

Figure 4. Position stage.

remotized and grounded actuation, leading to a third transmission subsystem. Each of them are now discussed in turn.

\subsection{Positioning Stage}

A line drawing view of the positioning stage connected to the distal stage enclosed in a protective cover (discussed in the next section) is shown on Figure 4. It is built around a four-bar mechanism which achieves static and dynamic balancing as well as minimization of inertia. The concept is inspired by the design of turntable tone-arms, actually subject to similar constraints. Consider

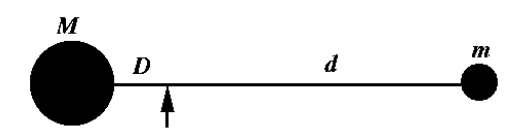

Figure 5.

Figure 5: $m$ refers to the mass of a cantilevered structure set at distance $d$ from a pivot point. Parameters $m$ and $d$ are given by geometrical and structural requirements. Find $M$ and $D$ such that the system is balanced under gravity and such that inertia is minimized. Static balancing yields $m d=M D$. Find $M$ such that $I=M D^{2}+m d^{2}=m d^{2}(m / M+1)$ is minimum. This expression is minimum when $M$ is maximum which means that the best design has the heaviest counter-weight. The mass is assumed to be concentrated at the motors, so we will use them as counter-weights. The first requirement is to place the motors so that the center of mass of the system coincides with its support center and the second is to keep this property invariant under any motion of the device. 
This led to what is shown on Figure 4. Motor-1 is fixed to the ground to produce a force at the tip principally in the $\mathrm{Y}$ direction (the sweep of joints is limited to $\left.\pm 30^{\circ}\right)$. Motor-2 drives four-bar regional structure, producing a force principally in the $\mathrm{Z}$ direction. It is placed to balance exactly the gravity contribution of the four bar structure around the axis of motor-1. Motor3 actuates the four-bar, producing a force principally in the $\mathrm{X}$ direction. The center of mass of a four-bar mechanism travels on a circle. If the circle vanishes to point, once balancing is achieved for one position, it is achieved for all. The center of mass of the four-bar is placed by design on the axis of motor-2, symmetrically to the center of mass of motor- 2 with respect to axis of motor- 1 . Thus, static balance is invariant with position.

The offsets of motor- 2 and motor- 3 are similar, so the inertia experienced at the tip is also similar in the $\mathrm{X}, \mathrm{Y}$, and $\mathrm{Z}$ directions. The design thus follows the "tone-arm rule" far all directions. All joints have a yoke design and are assembled throughout with pre-loaded bearings to guarantee absence of backlash even under conditions of high speed oscillatory motions. All parts are symmetrical so the Freedom-7 can be assembled in "righty" or in "lefty" configuration.

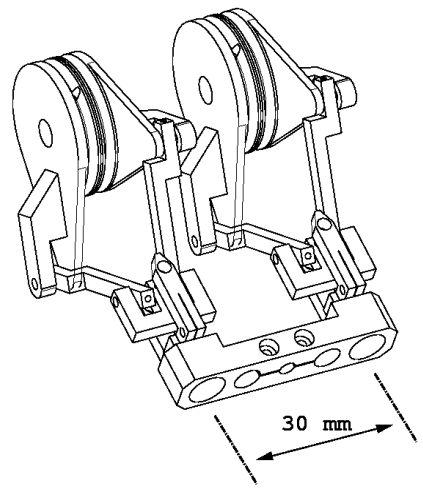

Figure 6. Distal orienting mechanism.

\subsection{Orienting Stage}

The mechanism provides four degrees of freedom, yet is relatively simple. It has a total of 15 parts which averages to only 4 per degree of freedom. An important consideration is made here: when mechanisms are scaled down, the angular work-space remain invariant. There are two limits to down-scaling in the present case: manufacturing and preservation of the sliding motion. A characteristic length (average length of moment arms) of about $30 \mathrm{~mm}$ was found to be best. At that small scale, the mechanism can be made quite light (less than $50 \mathrm{~g}$ ) for good structural properties even with conventional materials.

A line drawing view of the orientation stage is shown on Figure 6. It comprises two five bar linkages driven by twin pulleys and connected to the driven member by twinned spherical joints. The twin pulleys are supported by bearings (not shown) which allows them to swivel. The driven member clamps 
to an output rod (not shown) which is constrained to four degrees of freedom by a gimbal. Its last joint is a cylindrical pair. When the sliding motion is constrained this mechanism has three degrees of orientation freedom. In all cases, it preserves high kinematic conditioning throughout its work-space which is limited to $90^{\circ}$ of pitch, $100^{\circ}$ of yaw combined with a roll motion of $120^{\circ}$ in the present application. When not constrained, the differential sliding motion occurs when the two five bars deform identically. As an added bonus, a handle of standard size made of lightweight material will keep the orientation stage statically balanced. Moreover, because the positioning stage applies acceleration at the center of mass of the distal assembly, dynamic cross coupling terms are also minimized.

\subsection{Transmission}

In [3], the relative merits of transmission techniques for haptic interfaces have been reviewed, including: linkages, flexible elements (cables, steel belts, or polymeric tendons), shafts plus gears, or fluid lines connected to bellows. It was concluded that since transmissions work by exposing structural elements to stress, the best results are obtained when the largest amount of material is exposed to the most uniform stress, which led us to consider linkages or tendons. For reasons of bulk and complexity, the use of linkages should be limited to low numbers of degrees of freedom. In the case at hand, linkages provide for transmission of the positioning stage. In the orientation stage, linkages transmit motion from the four driven pulleys to the differential output interface. The motors of the orientations are all grounded and motion is transmitted to the orientation stage via high modulus polymeric tendons. The principal disadvantages of polymeric tendons over cables or steel belts is creep under permanent loading, and higher dissipation when routed around idlers. On the other hand, idlers diameters can be made small without causing fatigue. A tensioning technique has been devised for keeping the tendons under

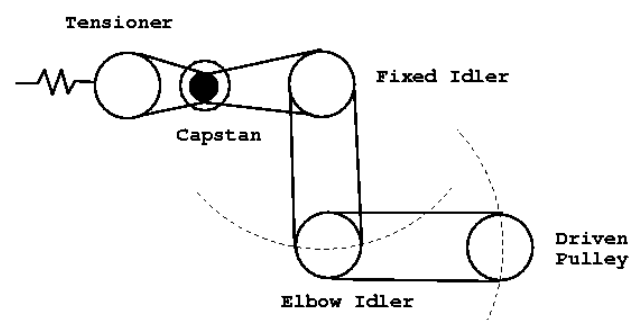

Figure 7. Transmission structure.

low stress when not transmitting torque. This reduces fatigue and long term creep. Its principle is shown on Figure 7. The routing is integrated within the position stage so that the tendon path lengths remains constant to a very small approximation ( $0.1 \%$ of their length) under any motion of the end effector. Sensing is combined with the tensioning pulley so that even if slip accidentally occurs at the capstan, the device remains calibrated. Because the actuators are grounded, the tendon path length is constant, and idlers and driven pulleys 
all have the same diameter, all kinematic and dynamic couplings between the two stages are eliminated.

\subsection{Sensors and Electronics}

All sensors are non-contact hall-effect angle transducers which guarantee high resolution and low noise. Signal conditioning as well as motor current drivers are presently linear electronics. They are also co-located which benefits closed loop control. As a bonus, no electric signal need to be transmitted beyond motor-3 which simplifies assembly, reduces noise and promotes reliability.

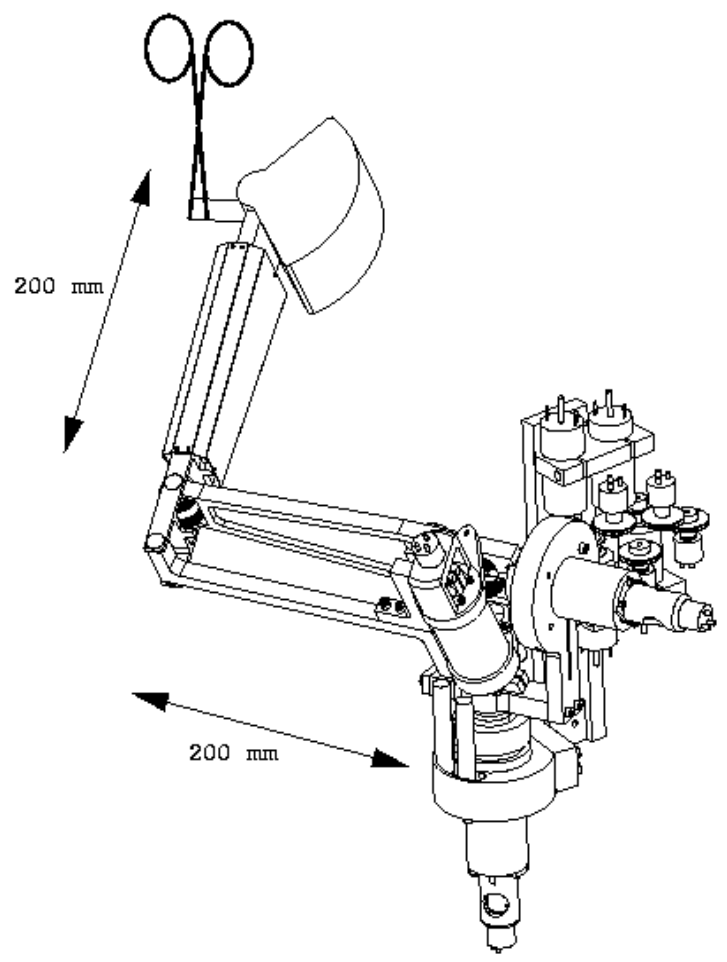

Figure 8. Freedom-7 shown without its holding stand.

\section{Results}

Figure 8 shows the complete integrated device without its table-top holding stand. The stand includes a clamping gimbal which permit the device to be positioned at any height and oriented in any pose with respect to the user. The figure also shows also the four motor-sensor-tensioner assembly which is tightly packaged and placed not to interfere with the device's workspace.

At the time of writing of this paper the construction of the described device is not completed so the results report on the performance of an available engineering prototype. Dynamic performance figures are well below the expected performance of the device currently being assembled. Actual figures will be available for the final version of this paper. 
A subset of the guidelines outlined in [13] is being followed:

Work-space: The device is wrist partitioned. Its workspace is limited by mechanical stops to its most dextrous region. The link lengths (200 mm each) and the mechanical stops have been optimized so that the translation workspace contains an ellipsoidal volume of axis lengths $130 \times 160 \times 180 \mathrm{~mm}$. The angular workspace is $90^{\circ}$ of pitch, $100^{\circ}$ of yaw combined with a roll motion of $120^{\circ}$. It's origin is not changed by more than $\pm 15^{\circ}$ throughout. These figures target the manipulation of tools with full wrist motions and elbow resting. The seventh differential output range is $10 \mathrm{~mm}$.

Intrusion: The device has the general appearance of an "elbow manipulator". The secondary link has a box-beam structure $(200 \mathrm{~mm}$ long, $30 \times 50 \mathrm{~mm}$ in section). The distal stage is enclosed in a protective case with section $50 \times 50$ $\mathrm{mm}$ and length $80 \mathrm{~mm}$. The case follows the angular motions of the output handle and may be used as a handle.

Output and Input Dynamic Range, Inertia: The mechanical noise of the device was measured under conditions of low velocity. A calibrated load-cell (Transducer Techniques model MDB-2.5/conditioner 308 calibrated at $\pm 10.0 \mathrm{~N}$ ) was set on the table of a milling machine and the device slowly back driven. A level of about $0.06 \mathrm{~N}$ of friction was measured in translation and $810^{-3} \mathrm{Nm}$ in angular motion. The short term peak force and torque are $5 \mathrm{~N}$ and $0.6 \mathrm{Nm}$. This corresponds to about one part in 1000 of dynamic range in translation and one in 100 in rotation. The position sensing resolution was also measured by driving the device back by known distances and was found to be better and $0.02 \mathrm{~mm}$. The inertia was measured. Known torques were applied and the resulting accelerations measured (Analog Device ADXL05 evaluation board). It was found to be $150 \mathrm{~g}$ in the $\mathrm{Y}$ direction and $90 \mathrm{~g}$ in the other two. The angular inertia at the handle is of the order of $0.1 \mathrm{gm}^{2}$.

To develop an intuitive idea of why that level of inertia is targeted, suppose that during a simulation an operator moves an instrument between two locations $10 \mathrm{~cm}$ apart in a one second motion. A symmetrical parabolic time optimal trajectory will require 0.5 second to accelerate and 0.5 second to stop, which yields an acceleration of $.4 \mathrm{~ms}^{2}$. The spurious inertial forces introduced by the device are then $.04 \mathrm{~N}$, a small number indeed, similar to the friction level of the device.

Frequency Response The frequency response was measured under two loading conditions: under isometric condition with the load cell, and under isotonic condition with the accelerometer (using a DSP Technology SIGLAB Model 20-22 signal analyzer).

Again, the reported results are those of the engineering prototype available at the time of the writing. Some structural weaknesses were found and corrected in the improved design. The response in $\mathrm{X}$ reveals a typical twomass resonance/antiresonance at $20 \mathrm{~Hz}$ which is attributed to a weakness in the secondary link. The response in $\mathrm{Y}$ has a smooth roll off after $30 \mathrm{~Hz}$. This was traced to lack of rigidity in the motor-1 connection. The angular response shows excellent results given that it has remotized actuation. The isotonic 

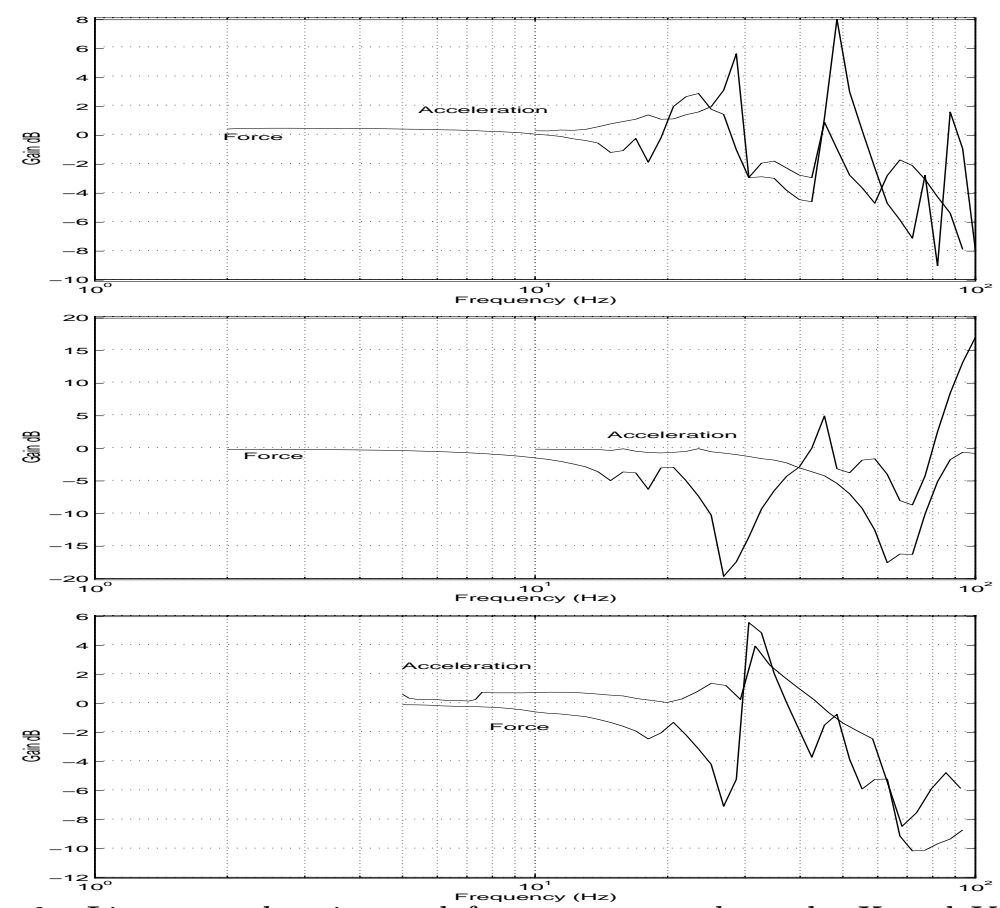

Figure 9. Linear acceleration and force response along the $\mathrm{X}$ and $\mathrm{Y}$ directions (top two plots). Angular acceleration and torque response around the $\mathrm{Z}$ direction (bottom plot).

acceleration response has a $\pm 3 \mathrm{~dB}$ response up to $50 \mathrm{~Hz}$, and was shown to transmit significant energy beyond $200 \mathrm{~Hz}$. The isometric response is more resonant and rolls off faster, as it is to be expected, due to the elasticity of the tendons. It is nevertheless considered excellent for a device of that size. Since sensing is co-located, it is possible to contemplate open loop frequency response shaping to extend the bandwidth without compromising the closed loop performance. Such a technique is however not as easily applicable to the metallic linkages of the positioning stage due to their sharp resonances, until they are replaced by better materials.

\section{Present and Future Construction}

The Freedom- 7 is presently made of aluminum for prototyping, however, this material has many limitations in this application. Research is under way to explore the use of advanced composite materials for the fabrication of haptic devices. Some preliminary results are available [14]. Studies are conducted on finite elements models in conjunction with the fabrication of prototype links for experimentation. These studies indicate that is possible to simultaneously raise the natural frequency and decrease the weight of these links. It is also possible to tailor other properties such as structural damping. With conventional construction methods, these requirements oppose each other. A view of the Freedom-7 composite construction is shown on Figure 10. 


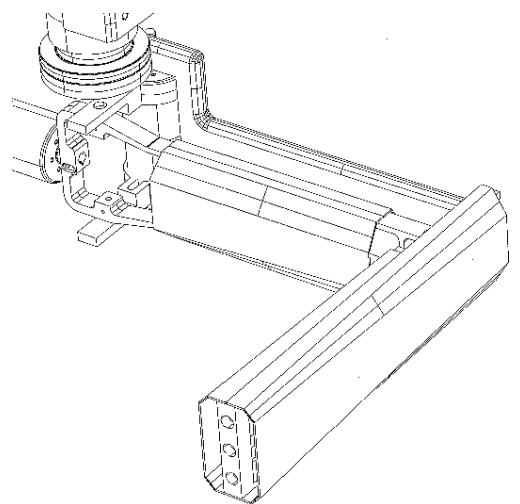

Figure 10. Advanced composite construction under development.

\section{Kinematics, Control and Computer Interface}

The coordinate transformations for position, velocity, and force admit closed forms for all seven degrees of freedom in the forward and inverse directions. When only the six first freedoms of the Freedom-7 are needed, actuators torques are calculated without having to resort to posing the problem as overconstrained: the output differential force is simply set to equal zero. Their implementation indicate that closed loop control in Cartesian coordinates can be achieved at a rate greater than a $\mathrm{KHz}$ on a Pentium class computer.

A computer control interface has been written to provide access to the device in Cartesian coordinates. The interface includes also sensor calibration functions and safety monitors that make sure that the device remains inside its thermal operational envelop.

Finally, the device has been included the library of devices supported by Armlib a package developed at the University of North Carolina [15] which facilitates the use of haptic devices in graphical applications.

\section{Acknowledgments}

Initial funding for this research was provided by the project "Haptic Devices for Teleoperation and Virtual Environments" (HMI-6) supported by IRIS (Phase 2 ), the Institute for Robotics and Intelligent Systems part of Canada's National Centers of Excellence program (NCE), and an operating grant "High Performance Robotic Devices" from NSERC, the National Science and Engineering Council of Canada. Additional funding for this research is provided by an NSERC University/Industry Technology Partnership Grant, McGill/MPBT "A Balanced Haptic Device for Computer/User Interaction". 


\section{References}

[1] Satava, R., M. 1996. Advanced simulation technologies for surgical education. Bull. A. Coll. Surg., 81(7):77-81.

[2] Kuppersmith, R. B., Johnston, R., Jones, S. B., Herman A. J. 1996. Virtual reality surgical simulation and otolaryngology, Arch. Otolaryngol. Head Neck Surg., 122:1297-1297.

[3] Hayward, V. 1995 (August, Pittsburgh). Toward a seven axis haptic interface. IROS'95, Int. Workshop on Intelligent Robots and Systems. IEEE Press, Vol. 2, pp. 133-139.

[4] Brooks Tigue, S. M. 1994. Instrumentation for the Operating Room: A Photographic Manual. Fourth edition. Toronto:Mosby.

[5] Kurtz, R., Hayward, V. 1992. Multi-goal optimization of a parallel mechanism with actuator redundancy. IEEE Transactions on Robotics and Automation. Vol. RA-8, No. 5. pp. 633-651.

[6] Goertz, R. C. 1952. Fundmentals of General Purpose Remote Manipulators. Nucleonics, 10(11), pp. 36-42.

[7] Vertut, J. 1976. Advance of the new MA 23 force reflecting manipulator system. Proc. 2nd International Symposium on the Theory and Practice of Robot and Manipulators, CISM-IFToMM, pp. 307-322.

[8] Bejczy, A. K., Salisbury, K. 1980. Kinesthetic coupling between operator and remote manipulator. Proc. International Computer Technology Conference, ASME, San Francisco, pp. 197-211.

[9] Asada, H., Yousef-Toumi, K. 1987. Direct Drive Robots: Theory and Practice MIT Press.

[10] Takase, K. 1984. Design of torque controlled manipulators composed of direct and low reduction ration drive joints. In Robotics Research: The First International Symposium, M. Brady and R. Paul (eds.). MIT Press, pp. 655-675.

[11] Massie, T. H., Salisbury, J. K. 1994. The Phantom haptic interface: a device for probing virtual objects. Proc. ASME Winter Annual Meeting, Symposium on Haptic Interfaces for Virtual Environment and Teleoperator Systems.

[12] Hayward, V., Choksi, J. Lanvin, G. Ramstein, C. 1994. Design and multiobjective optimization of a linkage for a haptic interface. In Advances in Robot Kinematics. J. Lenarcic and B. Ravani (Eds.). Kluver Academic. pp. 352-359.

[13] Hayward, V. Astley, O.R. 1996. Performance measures for haptic interfaces. In Robotics Research: The 7th International Symposium, Giralt, G., Hirzinger, G., (Eds.), Springer Verlag. pp. 195-207.

[14] McDougall, J., Lessard, L. B., Hayward, V. 1997, Applications of advanced materials to robotic design: The Freedom-7 haptic hand controller, to appear in the Proc. of 11th Int. Conf. on Composite Materials, Gold Coast, Australia, July 1997.

[15] Mark, W.R., Randolph, S.C., Finch, M., Van Verth, J.M., Taylor, R.M. 1996. Adding force feedback in graphics systems: issues and solutions, Computer Graphics Proceedings, Annual Conference Series, pp. 447-452. 